\title{
Variable modal parameter identification for non-linear mdof systems Part I: Formulation and numerical validation
}

\author{
Y.H. Chong and M. Imregun* \\ Imperial College of Science Technology and Medicine, \\ Mechanical Engineering Department, Exhibition \\ Road, London SW7 2BX, UK \\ Tel.: +44 207594 7068; Fax: +44 207584 1560; \\ E-mail: y.h.chong@ic.ac.uk andm.imregun@ic.ac.uk
}

Received 6 June 1999

Revised 5 January 2000

This paper deals with the formulation of a frequency domain modal analysis technique that is applicable to weakly non-linear multi-degree of freedom (MDOF) systems with well-separated modes. The concept of linear modal superposition is combined with the normal non-linear mode technique, an approach that allows the formulation of a system identification procedure in terms of variable modal parameters. The numerical study was focused on a 4-DOF system with cubic stiffness non-linearity, and the modal parameters were obtained as functions of the modal amplitude. It was shown that the methodology was well suited to the study of practical cases for which the underlying linear model may be approximate. Similarly, the technique was found to be robust in the presence of measurement noise, though some adverse effects were observed for high noise levels. Once the variable modal parameters were extracted at some given force level, the non-linear responses were predicted at other force levels via synthesis of normal non-linear modes. The same responses were also obtained using a harmonic balance approach and very good agreement was obtained between the two sets of results. The procedure is well suited to the study of industrial cases because of its compatibility with existing finite element methods and linear modal analysis techniques.

\section{Introduction}

Currently, three representations of the same model, namely response, spatial and modal descriptions, are

\footnotetext{
*Corresponding author.
}

routinely used for the prediction of the dynamic behaviour of a given structure. For linear systems, these representations are interchangeable and the choice of a particular description is based on the objectives of the analysis or the required form of the output. Spatial models are usually built for modelling purposes only and they have no direct established use in experimental modal analysis. Measured and predicted results can be compared using response models, though modal models are usually a more popular choice. Their widespread use stems from their compactness: a system with a large number of DOFs can be represented by a small number of modal parameters. In addition, they provide direct information about the actual mode shapes which are often of primary interest for minimising dynamic stresses. Consequently, most experimental parameter estimation techniques have been developed around modal models using the basic assumption of linearity. When dealing with non-linear systems, linear modal analysis techniques can be shown to introduce errors, usually by yielding complex mode shapes as if the system was non-proportionally damped [5]. Indeed, for situations involving large-amplitude vibration or significant nonlinearities, linear modal analysis tools are generally considered to be inadequate for obtaining reliable and consistent results. In such instances, the modal parameters may vary significantly with structural response, a feature that violates the principle of linearity.

Although several approaches have been proposed to provide qualitative and quantitative assessments of structural non-linearities, these are often limited to specific types and there are no techniques that can routinely deal with the general case $[4,13]$. Of particular interest here are the non-linear system identification tools and these can be classified as physical, non-physical, parametric and non-parametric. While a physical parameter based approach has the advantage of direct interpretability, it usually requires an explicit spatial model which can be very large for representative engineering systems. Such models are best suited to small systems, 
or perhaps to those with localised non-linearities. For large structures, modal models are often favoured over their spatial counterparts because of their computational efficiency in reducing bulky frequency domain data into a much smaller, albeit often truncated, set of eigen parameters. Thus, the idea of extending the use of linear modal models to the vibration analysis of non-linear systems is very attractive and such an approach will be pursued here.

The concept of a non-linear mode was first studied by [7] who used a geometrical method. His work initiated several studies which showed that the nonlinear normal mode concept could be used to study the behaviour of non-linear systems [10,14]. Using the same approach, [9] proposed a non-linear modal identification procedure which uses a non-physical, nonparametric formulation. Their work is compatible with existing linear techniques and makes use of the single non-linear resonant mode theory of $[11,12]$ and of equivalent linearisation approach of [2]. The analysis is based on a first-order frequency domain approximation and hence it requires much less computational effort than equivalent time domain methods. A significant drawback is the requirement to know the vibration characteristics of the underlying linear system prior to the analysis. However, in most cases, the use of low excitation levels may allow to obtain linearised FRFs for the non-linear system under study. A standard modal analysis of these low-excitation FRFs will then yield the underlying linear system. Such a route will also be explored here by extending the work of [9] to large MDOF systems representing real engineering structures, and by including both inherent structural damping and non-linear friction damping. It will also be shown that approximate underlying linear models will be sufficient for most engineering applications.

The main purpose of Part I is to describe a nonlinear modal analysis methodology that is applicable to weakly non-linear MDOF systems with well-separated modes and to validate the formulation using a 4-DOF numerical test case. The effects of wrongly-estimated linear underlying model and robustness in the presence of measurement noise will also be discussed in some detail. The experimental verification and the application to a representative engineering case will be considered in Part II. One of the advantages of the method is the ability to determine the response of the non-linear system at any force level once its variable modal parameters have been identified at some reference force level. Such a feature will allow to undertake more advanced applications such as the coupling of non-linear subcomponents.

\section{Background Theory}

The motion of an n-DOF non-linear system, subjected to a harmonic external force $\{\mathrm{F}\}$ with excitation frequency $\Omega$, can be described by:

$$
\begin{aligned}
& {[M]\{\ddot{X}\}+[C]\{\dot{X}\}+[K]\{X\}+\left\{f_{n l}(X, \dot{X})\right\}} \\
& =\{F\} \cos \Omega t
\end{aligned}
$$

where $[\mathrm{M}],[\mathrm{C}]$ and $[\mathrm{K}]$ are the mass, stiffness and damping matrices of the linear system, $\{\mathrm{X}\}$ is the physical response vector and $\left\{f_{n l}(x, \dot{x})\right\}$ is a non-linear restoring force vector which is a function of displacement and velocity. Linear modal analysis techniques assume that the non-linear term in (2) is small enough to be ignored so that the matrix equation can be decoupled using the eigenvectors of $[M]$ and $[K]$ matrices. However, such an approach is no longer applicable if the non-linear term, $\left\{f_{n l}\right\}$, is significant.

\subsection{Non-linear modal identification method}

While early single non-linear mode approaches accounted for natural frequency variations with the amplitude of the response, they ignored the amplitude dependence of the mode shapes. [11,12] argued that the use of a linear normal mode to describe the resonant condition of non-linear systems could lead to considerable discrepancies and suggested the use of the non-linear normal mode concept, introduced earlier by [7]. [9] assumed amplitude-dependent mode shapes and postulated that the combined effect of all non-linear contributions will only affect a selected single resonance of interest only, while the remaining modes will behave in a linear fashion. For example, if the excitation frequency, $\Omega$, is close to the $j^{t h}$ resonance, $\omega_{j}$, the relationship between modal co-ordinates and physical co-ordinates can be expressed as:

$$
\{X\}=\left[\{\phi\}_{1} \cdots\{\bar{\phi}\}_{j} \cdots\{\phi\}_{n}\right]\{Q\}
$$

where $\{Q\}$ is the modal response vector. The $j^{\text {th }}$ mode shape column, $\{\bar{\phi}\}_{j}$, is a function of the vibration amplitude, as indicated by the bar subscript. The other columns remain the same as the linear ones. Assuming that the non-linear mode shape, $\{\bar{\phi}\}_{j}$ is a linear combination of the linear resonant mode and its neighbours, one can write:

$$
\begin{aligned}
\{\bar{\phi}\}_{j}= & b_{j j}\{\phi\}_{j}+b_{1 j}\left(Q_{j}\right)\{\phi\}_{1}+b_{2 j}\left(Q_{j}\right)\{\phi\}_{2} \\
& +\cdots+b_{n j}\left(Q_{j}\right)\{\phi\}_{n} \\
= & \sum_{k=1}^{n} b_{k j}\left(Q_{j}\right)\{\phi\}_{k}
\end{aligned}
$$


where parameters $b_{k j}$ are the non-linear mode participation factors. These parameters are assumed to be a function of the resonant modal amplitude, $Q_{j}$. By definition, the diagonal terms, $b_{j j}$, are unity, while the off-diagonal terms, $b_{k j}$, are allowed to vary with the resonant modal amplitude, $Q_{j}$, and hence represent the coupled nature of the non-linear modal equations. In this way, it is possible to express all non-resonant modal displacement effects using a single resonant modal displacement term, a feature that will allow some decoupling of the full equations of motion. The coefficient $b_{k j}$ can also be written in the following form:

$$
\begin{aligned}
& b_{k j}\left(Q_{j}\right)=\{\phi\}_{k}^{T}[M]\{\bar{\phi}\}_{j} \\
& \forall Q_{j}: b_{j j}\left(Q_{j}\right)=1
\end{aligned}
$$

Clearly, the $b_{k j}$ matrix will be unity if the system is linear. Using (4), which defines the $j^{\text {th }}$ non-linear mode shape, (2) can be rewritten to yield the uncoupled equation of motion for the $j^{t h}$ non-linear mode. Pre and post multiplying the mass and stiffness matrices of (2) with the non-linear $j^{\text {th }}$ mode shape, the modal mass and stiffness matrices can be obtained as:

$$
\begin{aligned}
& {[\bar{m}]=[\bar{\phi}]^{T}[M][\bar{\phi}]}
\end{aligned}
$$

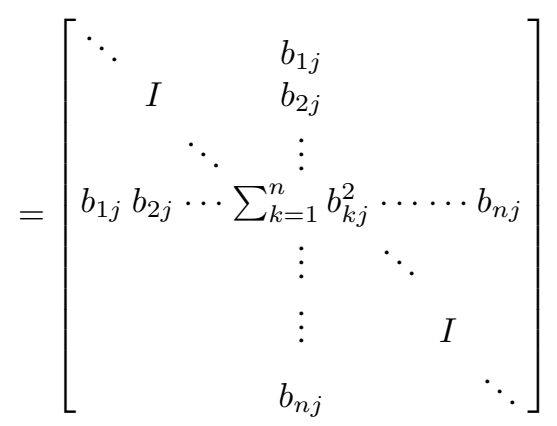

$$
\begin{aligned}
& {[\bar{k}]=[\bar{\phi}]^{T}[K][\bar{\phi}]=}
\end{aligned}
$$

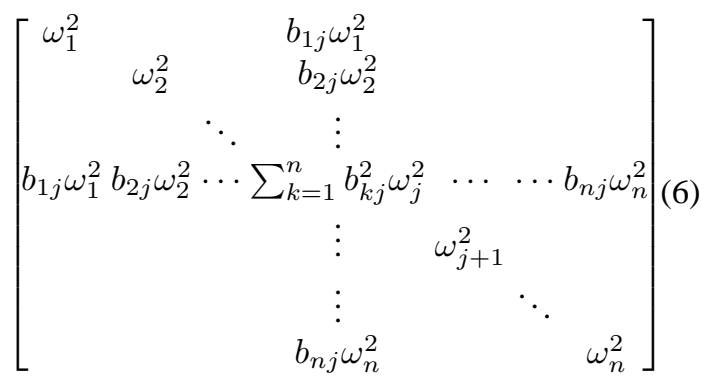

where, as before, the bar superscript represents the amplitude-dependent nature of the non-linear modal parameters. Although the normalised mass and stiffness matrices are not completely diagonal, the nondiagonal terms can be assumed to be small since the contribution of the non-resonant terms is small by definition. As an approximation, a non-linear modal stiffness correction term, $\bar{f}_{j}$, and a non-linear modal damping, $\bar{\xi}$, are introduced to the resonant modal equations to represent all such non-diagonal term contributions implicitly. The resonant modal equation of motion can then be written as:

$$
\begin{aligned}
& -\Omega^{2} \bar{m}_{j} Q_{j}+i \Omega \bar{\xi}_{j} Q_{j}+\left(\bar{k}_{j}+\bar{f}_{j}+i \eta_{j} \bar{k}_{j}\right) Q_{j} \\
& =\{\bar{\phi}\}_{j}^{T}\{F\}
\end{aligned}
$$

Equation (8) is identical to the original formulation by [9], except for the addition of a linear hysteretic damping term. The approximate non-linear modal mass and stiffness are given as:

$$
\begin{aligned}
& \bar{m}_{j}=\{\bar{\phi}\}_{j}^{T}[M]\{\bar{\phi}\}_{j} \approx \sum_{k=1}^{n} b_{k j}^{2} \\
& \bar{k}_{j}=\{\bar{\phi}\}_{j}^{T}[K]\{\bar{\phi}\}_{j} \approx \sum_{k=1}^{n} b_{k j}^{2} \omega_{j}^{2}
\end{aligned}
$$

Neglecting the damping term, (8) yields an approximate expression for the natural frequency of the $j^{t h}$ non-linear mode:

$$
\bar{\omega}_{j}^{2}=\frac{\bar{k}_{j}+\bar{f}_{j}}{\bar{m}_{j}}
$$

Upon substituting (9) into (8), the resonant modal displacement is given as:

$$
\begin{aligned}
Q_{j} & =\frac{\{\bar{\phi}\}_{j}^{T}\{F\}}{\left(\bar{\omega}_{j}^{2}-\Omega^{2}\right) \sum_{k=1}^{n} b_{k j}^{2}+i \eta_{j} \bar{k}_{j}} \\
& =\frac{\left(\sum_{k=1}^{n} b_{k j}\{\phi\}_{k}\right)^{T}\{F\}}{\left(\bar{\omega}_{j}^{2}-\Omega^{2}\right) \sum_{k=1}^{n} b_{k j}^{2}+i\left(\eta_{j} \bar{k}_{j}+\Omega \bar{\xi}\right)}
\end{aligned}
$$

\subsection{Synthesis of normal non-linear modes}

Using the single non-linear mode concept, the response of a non-linear system can now be computed as the summation of the non-linear contribution from the $j^{\text {th }}$ mode and linear contributions from the remaining modes. Around non-linear mode $j$, for excitation coordinate $i$ and response co-ordinate $m$, the non-linear receptance FRF $\left(\alpha_{i m}\right)_{N L}$ can be expressed as:

$$
\left(\alpha_{i m}\right)_{N L}=\left[\bar{\alpha}_{i m}\left(\Omega, Q_{j}\right)+\alpha_{i m}(\Omega)\right]
$$

where $\alpha_{i m}$, the linear part of the receptance FRF, can be written as:

$$
\begin{array}{r}
\alpha_{i m}(\Omega)=\sum_{l=1}^{n} \frac{\phi_{i l} \phi_{m l}}{m_{l}\left(\omega_{l}^{2}-\Omega^{2}\right)+i \eta_{l} k_{l}} \\
l \neq j
\end{array}
$$


The $j^{t h}$ non-linear modal contribution is given as:

$$
\begin{aligned}
\bar{\alpha}_{i m}\left(\Omega, Q_{j}\right) & =\frac{\overline{\phi_{i j}} \bar{\phi}_{m j}}{\bar{m}_{j}\left(\bar{\omega}_{j}^{2}\left(Q_{j}\right)-\Omega^{2}\right)+i\left(\eta_{j} \bar{k}_{j}+\Omega \bar{\xi}\right)} \\
& =\frac{\left(\sum_{s=1}^{n} b_{s j} \phi_{i s}\right)\left(\sum_{k=1}^{n} b_{k j} \phi_{m k}\right)}{\bar{m}_{j}\left(\bar{\omega}_{j}^{2}-\Omega^{2}\right)+i\left(\eta_{j} \bar{k}_{j}+\Omega \bar{\xi}\right)}(13)
\end{aligned}
$$

\subsection{Extension to non-linear modal parameter extraction}

The basic idea behind non-linear parameter extraction is to minimise the difference between measured (or simulated) receptances and those obtained from (11) by varying the non-linear parameters, $b_{k j}$ and $\bar{\omega}_{j}$. The problem can be solved numerically by using any standard multi-variable minimisation process. The size of the problem is determined by the number of participating neighbouring modes to be included in the analysis. For instance, if all non-linear parameters were to be included in the analysis, the number of parameters to be extracted would be the same as the number of DOFs. Numerical experience indicates that the $b_{k j}$ parameters which are not near the resonant mode of interest can be ignored and the analysis can be conducted by retaining a few neighbouring modes only. In any case, the identification process involves the use of a column of receptance matrix which contains the necessary information for determining the vibration characteristics of a structure. By separating the measured and predicted non-linear responses into their real and imaginary parts, the function to be minimised can be written as:

$$
\begin{aligned}
g_{R 1}\left(\bar{\omega}_{j}^{2}, b_{k j}\right)= & R E\left\{\alpha_{1 m}^{*}\right\}-R E\left\{\left[\bar{\alpha}_{1 m}\left(\Omega, Q_{j}\right)\right.\right. \\
& \left.\left.+\alpha_{1 m}(\Omega)\right]\right\} \\
\vdots & \\
g_{R n}\left(\bar{\omega}_{j}^{2}, b_{k j}\right)= & R E\left\{\alpha_{n m}^{*}\right\}-R E\left\{\left[\bar{\alpha}_{n m}\left(\Omega, Q_{j}\right)\right.\right. \\
& \left.\left.+\alpha_{n m}(\Omega)\right]\right\} \\
g_{I 1}\left(\bar{\omega}_{j}^{2}, b_{k j}\right)= & I M\left\{\alpha_{1 m}^{*}\right\}-I M\left\{\left[\bar{\alpha}_{1 m}\left(\Omega, Q_{j}\right)\right.\right. \\
& \left.\left.+\alpha_{1 m}(\Omega)\right]\right\} \\
\vdots & \quad I M\left\{\alpha_{n m}^{*}\right\}-I M\left\{\left[\bar{\alpha}_{n m}\left(\Omega, Q_{j}\right)\right.\right. \\
& \left.\left.+\alpha_{n m}(\Omega)\right]\right\}
\end{aligned}
$$

where the $g$ 's are the functions to be minimised and $\alpha^{*}$ 's are the measured receptances. One of the simplest approaches to minimising (15) is to monitor the norm of the vector $\{g\}$ which contains 2 n functions because of the explicit treatment of the real and imaginary parts. In the following numerical studies, the minimisation was carried out by a MATLAB subroutine which uses the Gauss-Newton method with a mixed quadratic/cubic line search procedure.

At the end of the minimisation process, each excitation frequency used yields a set of non-linear modal parameters which are associated with that particular modal amplitude. The non-linear mode shape is then obtained by inserting the identified participation factor, $b_{k j}$, and the (known) linear mode shape into (4). Using the calculated non-linear mode shape for the $j^{\text {th }}$ mode, the corresponding resonant modal amplitude, $Q_{j}$ is determined from the measured physical displacement amplitude by inverting (2). The variation of the non-linear modal parameters with the resonant modal amplitude can then be obtained by considering a sufficient number of excitation frequencies. Further analysis may require a suitable polynomial curve-fit to this variation so that a continuous representation can be used.

\section{Generation of reference data for non-linear response}

Using an equivalent linearisation technique, it is possible to use (1) to compute the non- linear response. Such an approach allows not only the generation of reference data for non-linear modal analysis but it also enables the validation of the resulting non-linear modal model by considering several force levels. The equivalent linearisation method is essentially an optimization process which seeks a linearised representation of a non-linear system at some defined amplitude so that the difference between the two states is minimised [2]. One of its best known variants, the harmonic balance method (HBM), determines the equivalent linear stiffness by calculating the harmonic terms of the non-linear force over one vibration cycle. Despite its approximate nature, its computational efficiency made the HBM a favoured choice over time-domain techniques or higher-order FRF representations [1]. Although some unsymmetrical non-linearities are known to exhibit significant super and sub harmonic characteristics, the fundamental harmonic is still much larger for a wide class of non-linearities, such as those studied here. In any case, the HBM has been checked through experiments and direct numerical integration [3,6,8]. Further experimental validation will be presented in Part II of this paper. 


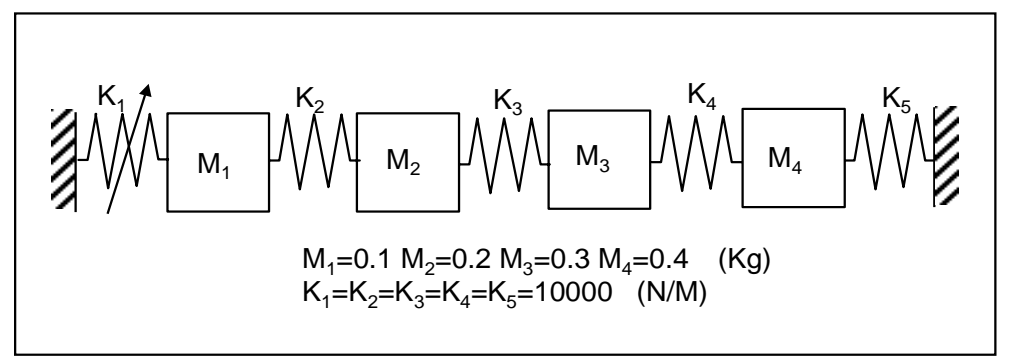

Fig. 1. The 4-DOF system.

Assuming harmonic oscillation and considering the first harmonic only, the real and imaginary parts of the linearised stiffness can be expressed as [8]:

$$
\begin{aligned}
& K_{r}^{\prime}(X)=\frac{1}{\pi X} \int_{0}^{2 \pi} f_{n l} \cos \Omega t d t \\
& K_{i}^{\prime}(X)=\frac{-1}{\pi X} \int_{0}^{2 \pi} f_{n l} \sin \Omega t d t
\end{aligned}
$$

where both integrals of $f_{n l}$ represent the fundamental harmonic of the non- linear force. These are normalised by the amplitude of response, $X$, to obtain the required describing functions. The above non-linear terms can then be inserted into (2) to calculate an amplitudedependent quasi-linear receptance matrix, $[\alpha(X)]$. For a system excited by external force $\{F\}$, the equation of motion can be written as:

$$
\{X\}=[\alpha(X)]\{F\}
$$

and solved iteratively, say using a Newton-Raphson approach.

\section{Case Study}

\subsection{4-DOF non-linear system}

The case study was conducted on a 4-DOF massspring system with $0.5 \%$ proportional structural damping. As shown in Fig. 1, a non-linear cubic stiffness element was introduced between the ground and first mass. The loading characteristic of the non-linear spring is given by $f_{n l}=\beta X^{3}$ and its describing function can be written as [6]:

$$
K^{\prime}(X)=\frac{3}{4} \beta X^{2}
$$

The severity of the non-linear element is determined by the coefficient of cubic term, $\beta$, and a value of 200 was used for the case study. Figure 2 shows the effect of cubic stiffness non-linearity on the response of the structure. One of the most distinctive features of this particular non-linearity type is the leaning of the resonance peak towards right. The distortions are mostly confined to the resonant regions and the deviation is greatest for the $4^{\text {th }}$ mode where the response amplitude of Mass 1 is largest. Thus the $4^{t h}$ mode was chosen for the proposed non-linear modal analysis.

\subsubsection{Analysis using all 4 modes}

As mentioned previously, the number of parameters to be extracted depends on the number of modes included in the analysis. In this particular example, the effects of all three neighbouring modes were included. Consequently, there are 4 non-linear parameters that need to be extracted: the non-linear natural frequency, $\bar{\omega}_{4}$, and three $b_{k j}$ parameters, namely $b_{14}, b_{24}$ and $b_{34}$.

The first column of the response matrix was obtained via HBM simulations. The response data were computed for a force level of $200 \mathrm{~N}$ applied at Mass 1. A non-linear modal analysis was performed next using the approach described in Section 2 and the findings are shown in Fig. 3. The stiffness increases with the response amplitude and this results in an increase in natural frequency, $\bar{\omega}_{j}$. As the non-linearity becomes more pronounced, the resonance tends to lean more and more to the right until a jump occurs, this being the result of a sudden large change in response due to a small perturbation. The point where the jump phenomenon occurs largely depends on the size of frequency step taken for the simulation. Also, depending on increasing and decreasing frequency steps, the "jump frequency" will be different. The results of the non-linear modal analysis in Fig. 3 concur with the characteristics described above. The hardening nature of the stiffness can be seen in the variation of the non-linear natural frequency, $\bar{\omega}_{4}$, with the modal amplitude. The participation of neighbouring modes, $b_{k j}$, also increase with the modal amplitude, indicating a greater distortion of the linear mode. Another noteworthy finding is the decrease of 


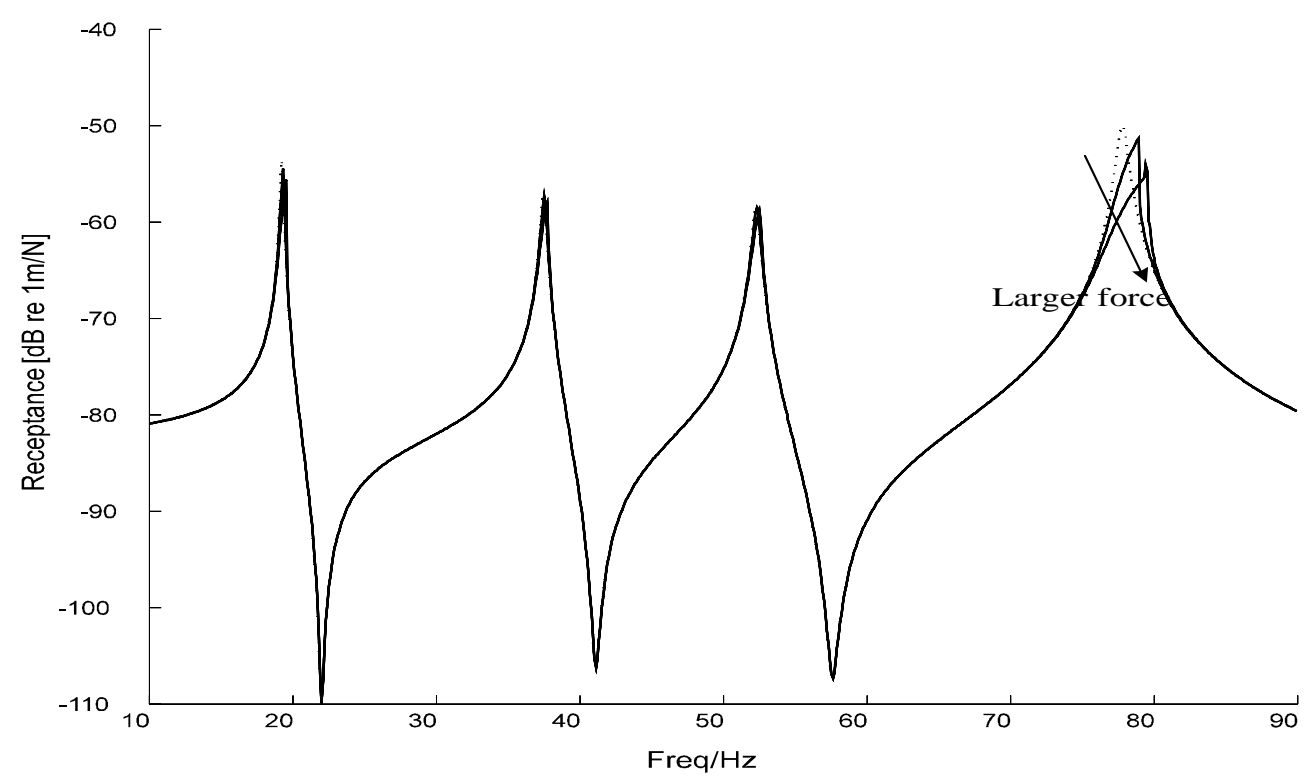

Fig. 2. Response of the 4 DOF system with cubic stiffness non-linearity.

$b_{k j}$ value as one moves away from the resonant mode, justifying that the corresponding modes can be ignored.

To complete the identification process, second-order polynomials were chosen to curve-fit the variation of the non-linear modal parameters (Fig. 3). The mathematical model of the non-linear modal parameters for the $4^{t h}$ mode in terms of the $4^{t h}$ modal amplitude, $Q_{4}$, is thus given by:

$$
\begin{aligned}
& \omega_{4}^{2}=c_{11} Q_{4}^{2}+c_{12} Q_{4}+c_{13} \\
& b_{14}=c_{21} Q_{4}^{2}+c_{22} Q_{4}+c_{23} \\
& b_{24}=c_{31} Q_{4}^{2}+c_{32} Q_{4}+c_{33} \\
& b_{34}=c_{41} Q_{4}^{2}+c_{42} Q_{4}+c_{43}
\end{aligned}
$$

where the $c_{i j}$ parameters are the actual polynomial coefficients. Each mode of the system will generate a similar set of parameters to represent the non-linear behaviour at that particular mode. For this example, the modal parameters of the first three modes may be expected to be more or less linear.

\subsubsection{Response generation at other force levels}

One of the major attractions of a non-linear modal model is the ability to predict the response at any force level. Here new responses were computed for force levels of $200 \mathrm{~N}, 160 \mathrm{~N}$ and $100 \mathrm{~N}$ using the modal parameters extracted for a force level of $200 \mathrm{~N}$. The regenerated and directly-computed responses are plotted in Fig. 4 for all three force levels. There is very good agreement between the two sets. Furthermore, typical non-linear stiffness characteristics, such as non-circular Nyquist plots and jump phenomenon are successfully preserved by the extracted non-linear parameters.

\section{Robustness checks}

\subsection{Sensitivity to the accuracy of the underlying linear model}

The underlying linear model of a non-linear system is usually obtained by exciting the system at a low force level and performing a linear modal analysis on the measured response functions ${ }^{1}$. It is also possible to use FE modelling for simple cases. However, neither approach can guarantee an accurate linear model and the consequences of having an approximate underlying linear model will be investigated here.

An approximate underlying linear model of the 4DOF system of Fig. 1 will now be obtained through response measurements taken at a relatively high force level of $100 \mathrm{~N}$. The results of a linear modal analysis, followed by response regeneration using the extracted linear modal parameters, are shown in Fig. 5 where the true linear response is also plotted. It is clearly seen that the linearised response is significantly different from the true linear response obtained from the exact underlying system. Nevertheless, the approximate underlying linear model, obtained from the linear modal analysis, was used as input data to a non-linear modal analysis. The results are shown in Fig. 6. Also

\footnotetext{
${ }^{1}$ Another way of linearising the response is to use the averaging properties of a random excitation signal, though the numerical simulation of such a route is not straightforward.
} 


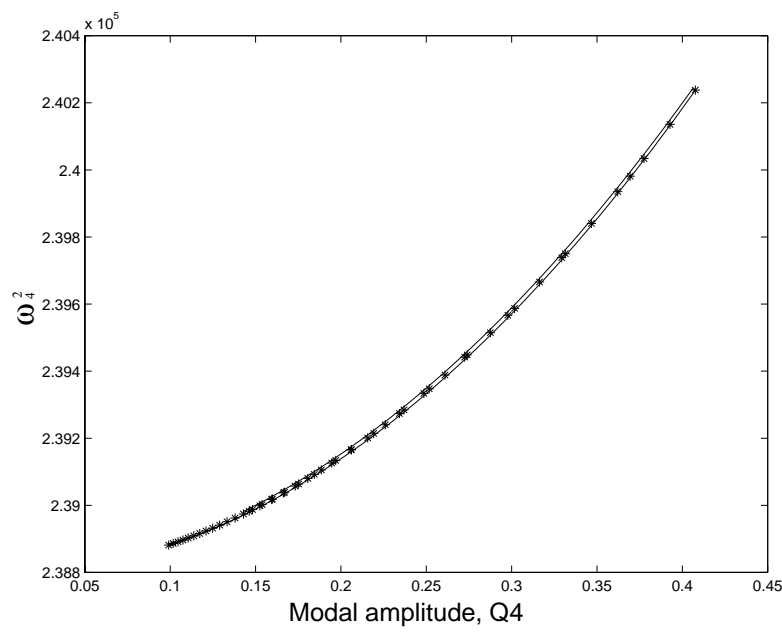

(a) Variation of $4^{\text {th }}$ natural frequency

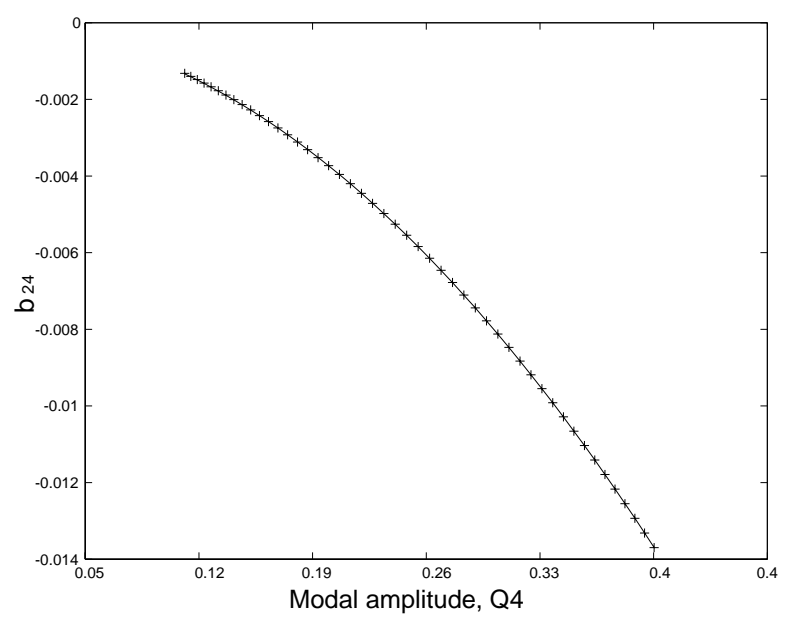

(c) Variation of $b_{24}$

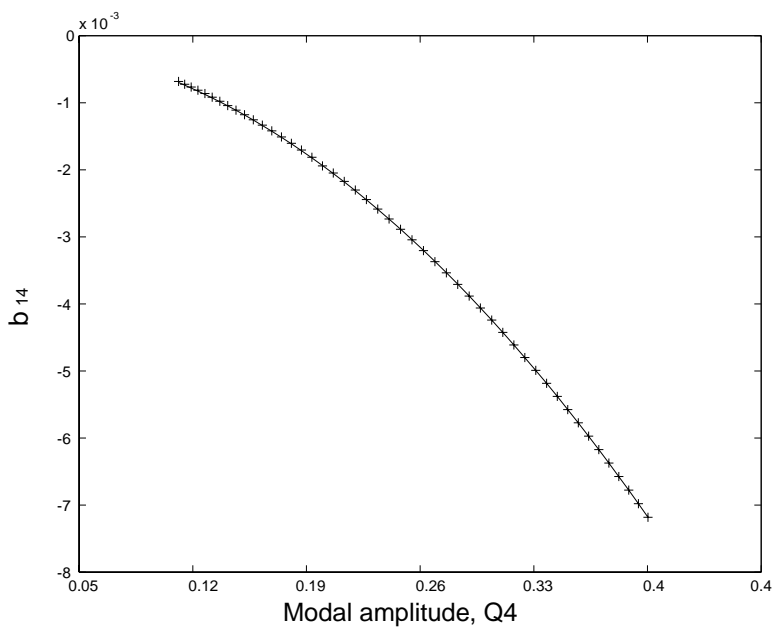

(b) Variation of $b_{14}$

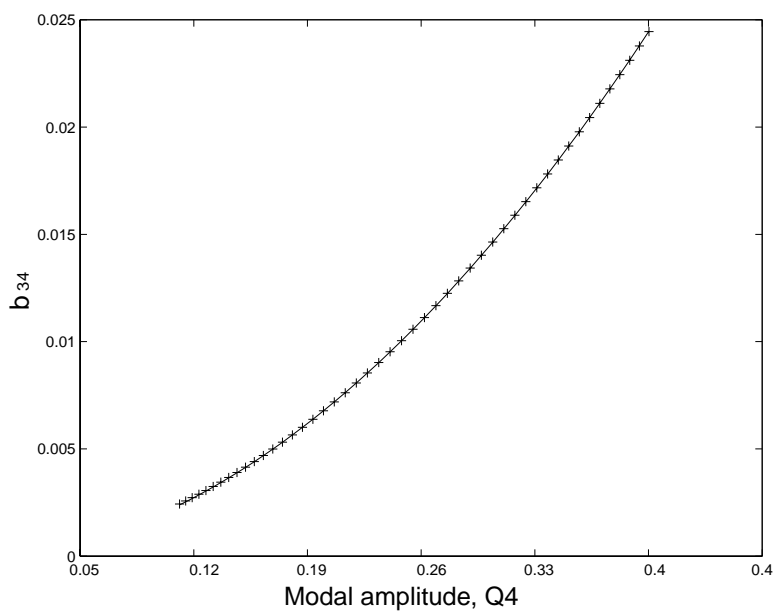

(d) Variation of $b_{34}$

Fig. 3. Non-linear modal parameter variations for the 4-th mode: $* * *$ Extracted values_-_ Curve-fitted values.

plotted in the same figure are the results obtained with the exact underlying linear model. It is easily seen that both sets of results are very similar, with the exception of the $b_{k j}$ parameter which shows an offset. On the other hand, the natural frequency variation is identical, indicating that approximate underlying linear models can be used in non-linear parameter identification. This observation has a significant implication for real applications since it is almost always possible to obtain an approximate linear system via a linear modal analysis of responses obtained at low forcing.

The curve-fitted non-linear modal parameters were used to regenerate responses at force levels of $1 \mathrm{~N}, 100 \mathrm{~N}$ and $200 \mathrm{~N}$. The same responses were also obtained via HBM simulations for comparison purposes (Fig. 7). Very good agreement was obtained in all cases, including the $1 \mathrm{~N}$ force level, which effectively corresponds to the true underlying linear response.

\subsection{Effects of measurement noise}

$5 \%$ random noise was added to the non-linear responses generated for a force level of $100 \mathrm{~N}$ and a linear modal analysis was carried out as before to obtain the underlying linear model. Using this approximate underlying linear model, a non-linear modal analysis was 


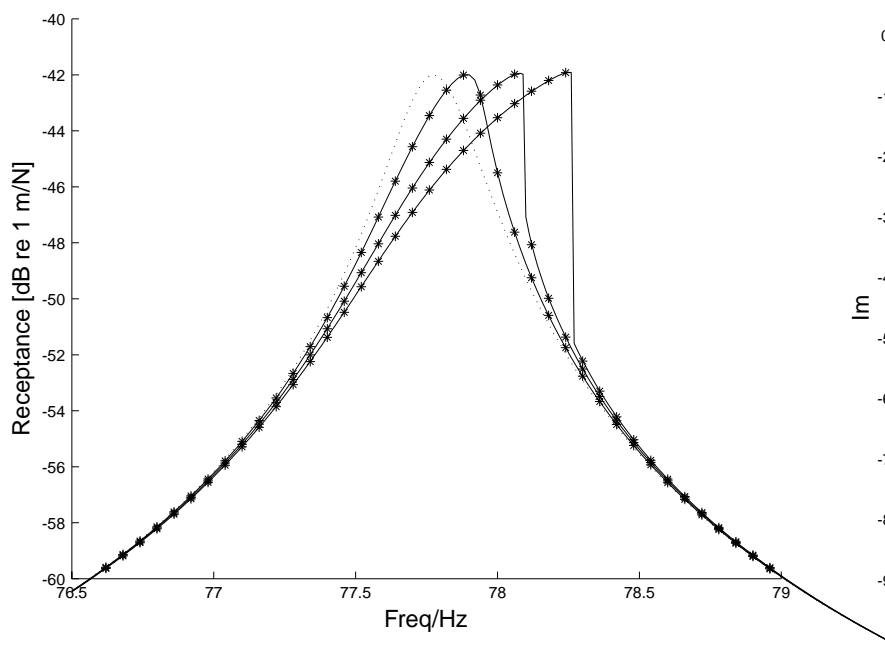

(a) Modulus plot

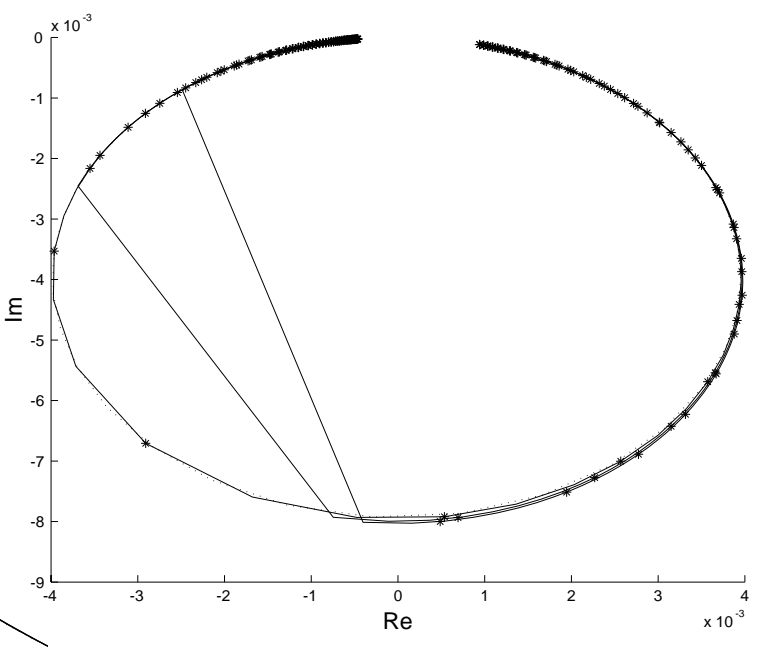

(b) Nyquist plot

Fig. 4. Response predictions with different force levels: . . Linear FRF,_- HBM simulation, $* * *$ Regenerated using modal parameters.

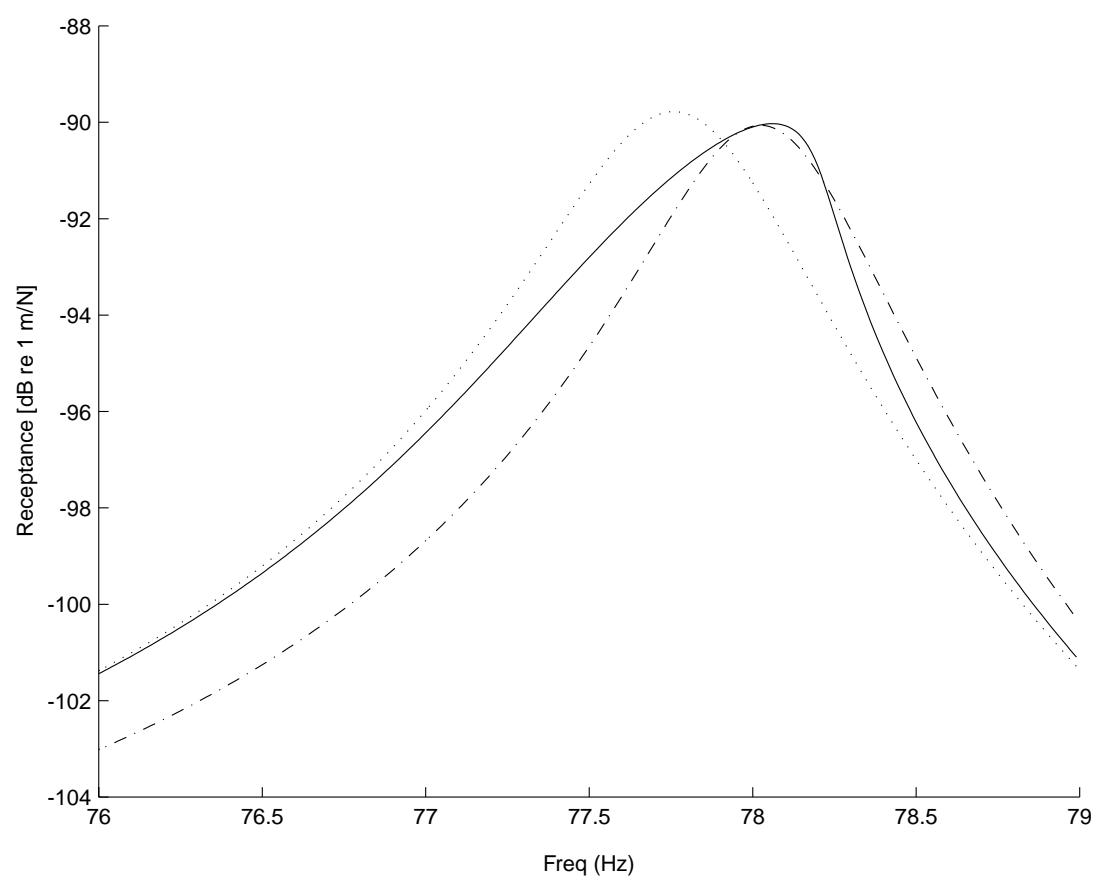

Fig. 5. Linear modal analysis of non-linear response: . . . True linear FRF, _ . _ . - Linearised response through linear modal analysis, $\_$True non-linear response.

conducted on the noise-added responses and the results are shown in Fig. 8. The non-linear modal parameter variations show a clear noise effect, though a curve-fit is relatively straightforward for the natural frequency variation (Fig. 8). Although it can be argued that the level of noise is unacceptable for curve-fitting the $b_{k j}$ parameter, the overall trend is well captured by a cubic polynomial, the order of which is selected using the natural frequency variation. In any case, experimental noise levels are usually around 1-3\% and hence a typical situation may well be less severe than that studied here. Finally, Figure 9 shows the response predictions for various force levels using the extracted non-linear modal parameters. There is good agreement between 


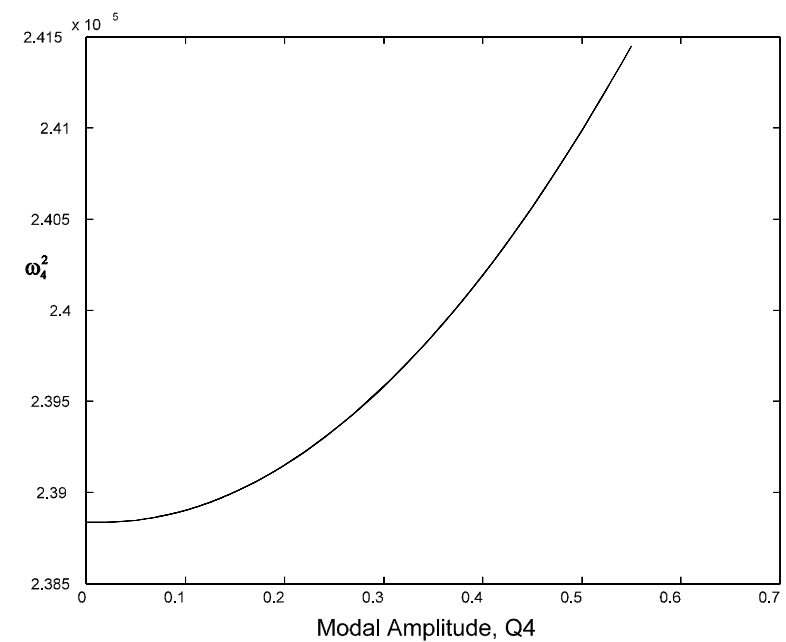

(a) Natural frequency variation for the $4^{t h}$ mode

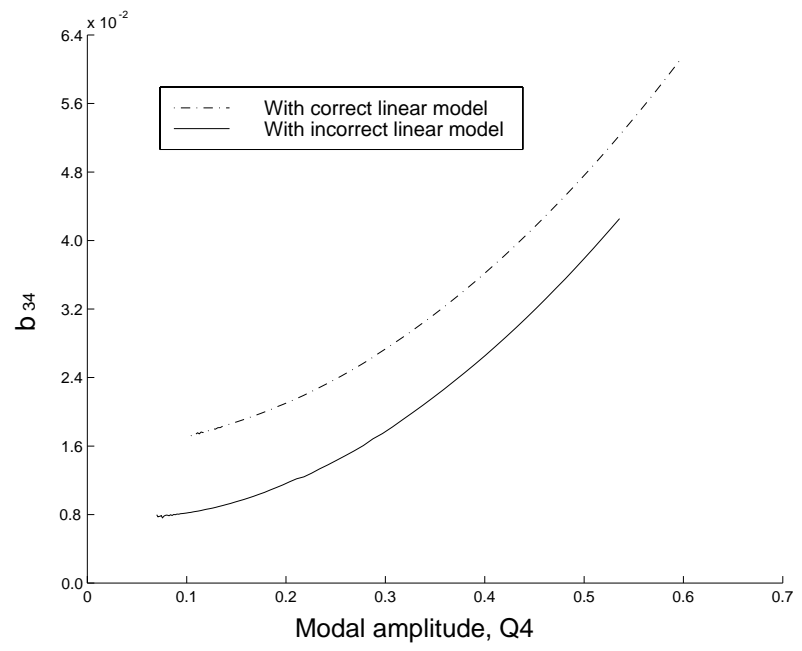

(b) Variation of $b_{34}$

Fig. 6. Non-linear modal parameters obtained with approximate and exact underlying linear models.

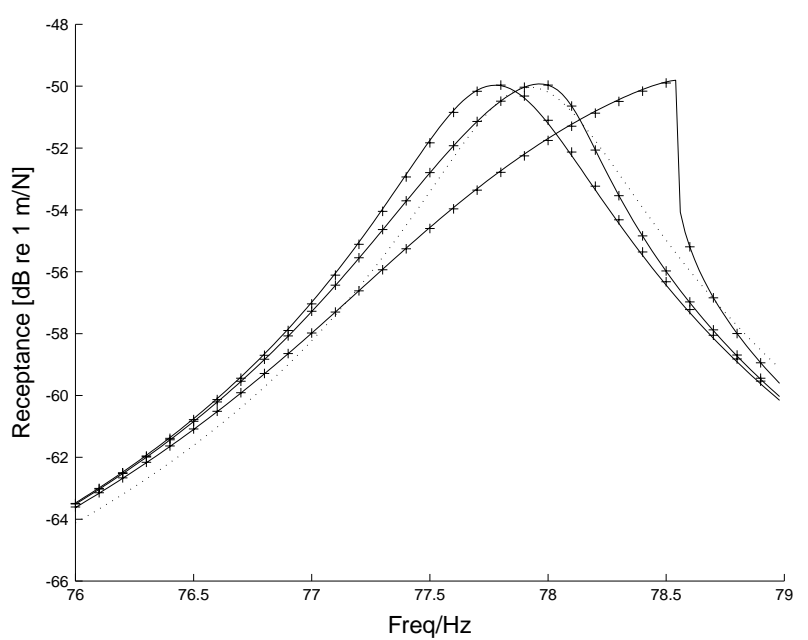

(a) Modulus plot

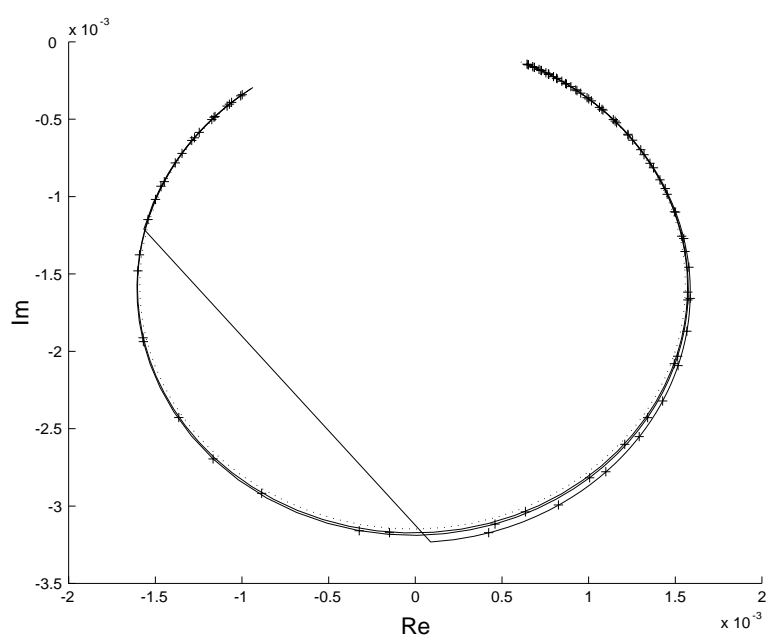

(b) Nyquist plot

Fig. 7. Response predictions with an approximate underlying linear model: ... Regeneration with linear modal parameters only,-- HBM simulations, ++ Regeneration with non-linear parameters.

direct simulations and the regenerated responses, a feature which indicates the robustness of the non-linear parameter identification technique in the presence of measurement errors.

\section{Concluding Remarks}

- A modal analysis method has been presented for weakly non-linear MDOF systems with well- separated modes. It has been shown that the technique is applicable to practical situations since it can cope with approximate underlying linear models and tolerate experimental noise.

- Once the non-linear modal parameters are obtained for a given force level, the response of the non-linear system was successfully predicted at other force levels, even in the presence of significant measurement noise. However, numerical tests show that the force levels for data acquisition 


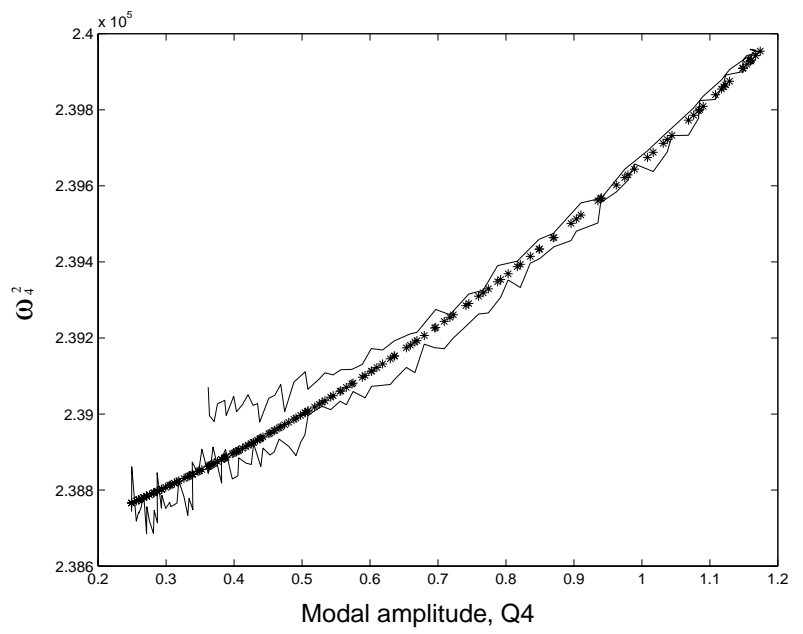

(a) Variation of $4^{\text {th }}$ mode resonant frequency

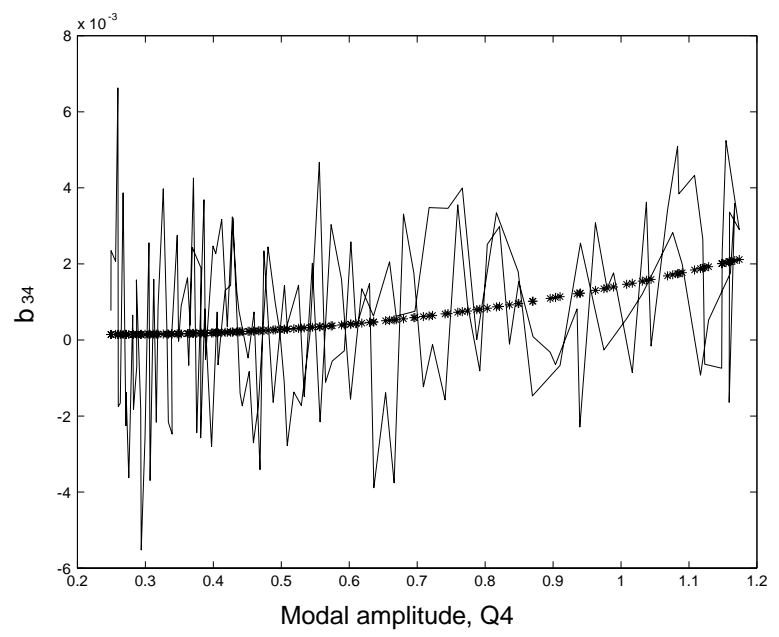

(b) Variation of $b_{34}$

Fig. 8. Noise effect on non-linear modal parameter extraction: — Extracted values, - - - Fitted values.

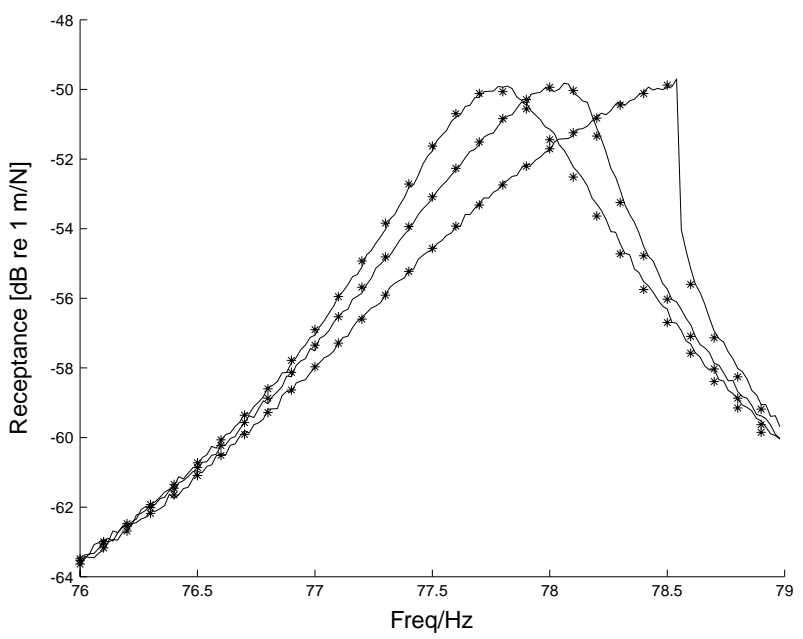

(a) Modulus plot

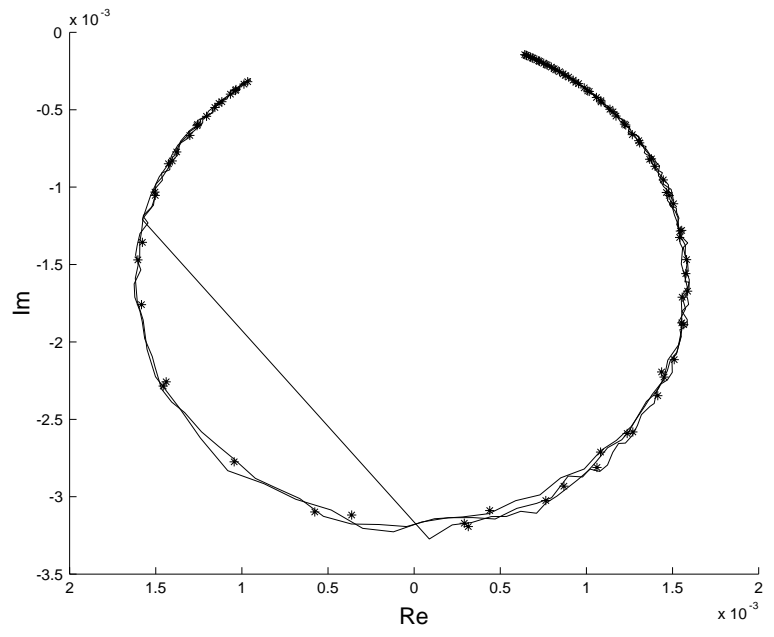

(b) Nyquist plot

Fig. 9. Regeneration from noise-polluted data: _- HBM simulation, $* * * *$ Regenerated FRF.

and response calculations must not be more than two orders of magnitude apart, though this feature is likely to be case dependent.

- The non-linear modal model can therefore be used to minimise experimental testing by enabling the analyst to obtain the response levels under a wide range of forcing conditions. It is also possible to perform a sub-structure analysis by coupling several non-linear components.

- Being based on a modal representation, the technique is particularly well suited to the study of large systems. Further numerical studies, not re- ported here, showed that only a few modes around the resonance of interest were needed to predict the non-linear contributions. This feature will be exploited when studying a large MDOF system in Part II.

\section{References}

[1] S.J. Gifford and G.R. Tomlinson, Recent advances in the application of functional series to non-linear structures, Journal of Sound \& Vibration 135(2) (1989), 289-317. 
[2] W.D. Iwan, A generalization of the concept of equivalent linearization, International Journal of Non-linear Mechanics 18 (1973), 149-165.

[3] K. Murakami and H. Sato, Vibration characteristics of a beam with support accompanying clearance, Journal of Vibration, Stress and Reliability in Design 112 (October 1990), 508-514.

[4] H.G. Natke, J.N. Juang and W. Gawronski, A brief review on the identification of non-linear mechanical systems, Kissimmee, Florida, Proc. of IMAC 6, 1988, pp. 1569-1574.

[5] H.N. Ozguven and M. Imregun, Complex modes arising from linear identification of nonlinear systems, International Journal of Experimental \& Analytical Modal Analysis 8(2) (1993), 151-164.

[6] H.N. Ozguven, B. Tanrikulu and M. Imregun, M. forced harmonic response analysis of non-linear structures using describing functions, AIAA Journal 31(7) (1993), 1313-1320.

[7] R.M. Rosenberg, The normal modes of non-linear $\mathrm{n}$ degreeof-freedom systems, ASME Journal of Applied Mechanics $\mathbf{8 2}$ (1962), 7-14.

[8] K.Y. Sanliturk, M. Imregun and D.J. Ewins, Harmonic balance vibration analysis of turbine blades with friction dampers, ASME Journal of Vibration \& Acoustics 118 (1996), 96-103.

[9] S. Setio, H.D. Setio and L. Jezequel, A method of non-linear modal identification from frequency response tests, Journal of Sound \& Vibration 153(3) (1992), 497-515.

[10] S.W. Shaw and C. Pierre, Normal modes of vibration for nonlinear continuous systems, Journal of Sound \& Vibration 2 (1994), 319-347.

[11] W. Szemplinska-Stupnicka, The modified single mode method in the investigations of the resonant vibrations of non-linear systems, Journal of Sound \& Vibration 63 (1979), 475-489.

[12] W. Szemplinska-Stupnicka, Non-linear normal modes and generalized ritz method in the problems of vibrations of nonlinear elastic continuous systems, International Journal of Non-linear Mechanics 18 (1983), 149-165.

[13] G.R. Tomlinson, Linear or non-linear - that is the question, Proc. ISMA 19, KU Leuven, 1992, pp. 11-32.

[14] A.F. Vakakis, Non-linear normal modes and their applications in vibration theory: an overview, Mechanical Systems \& Signal Processing 11(1) (1997), 3-22. 

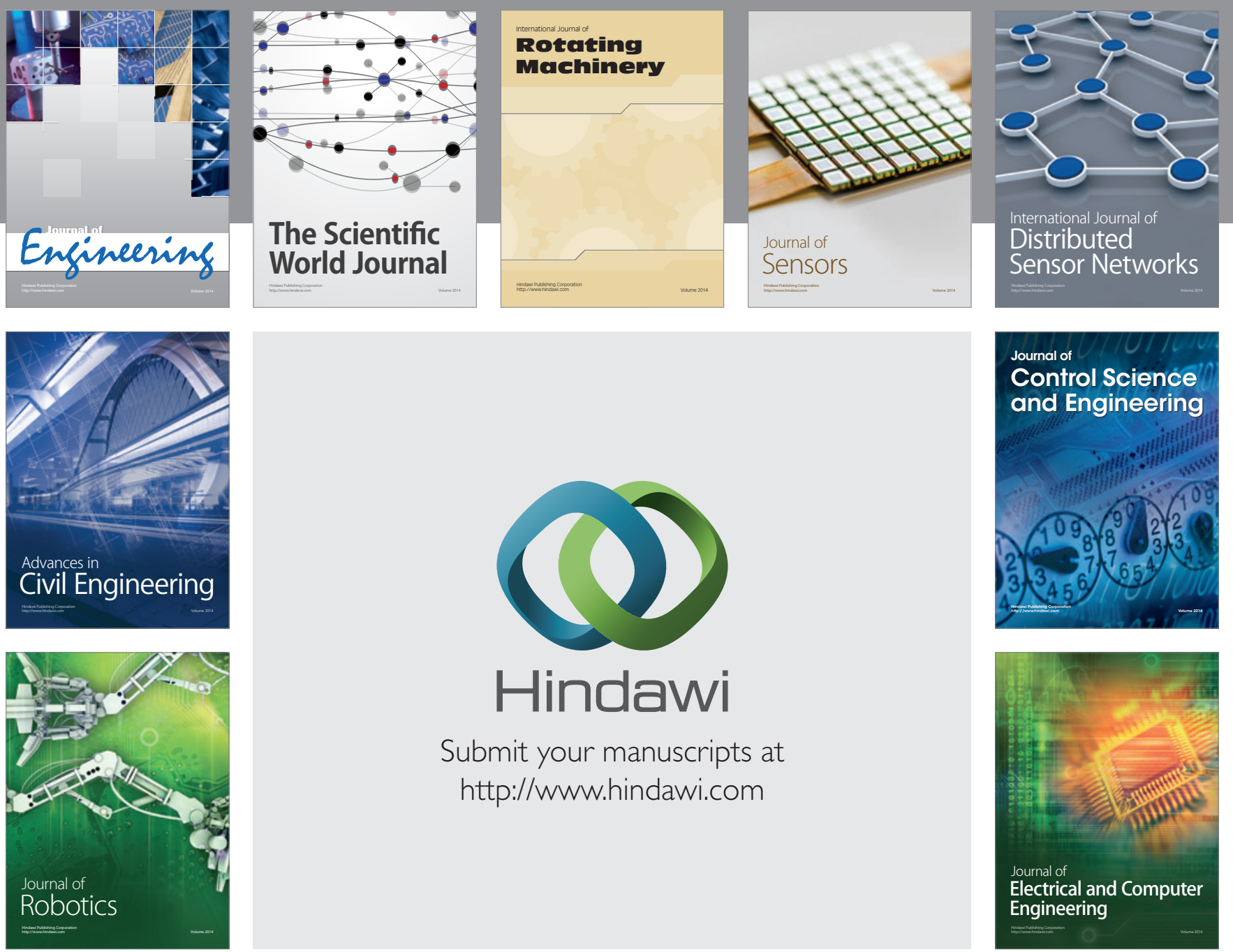

Submit your manuscripts at

http://www.hindawi.com
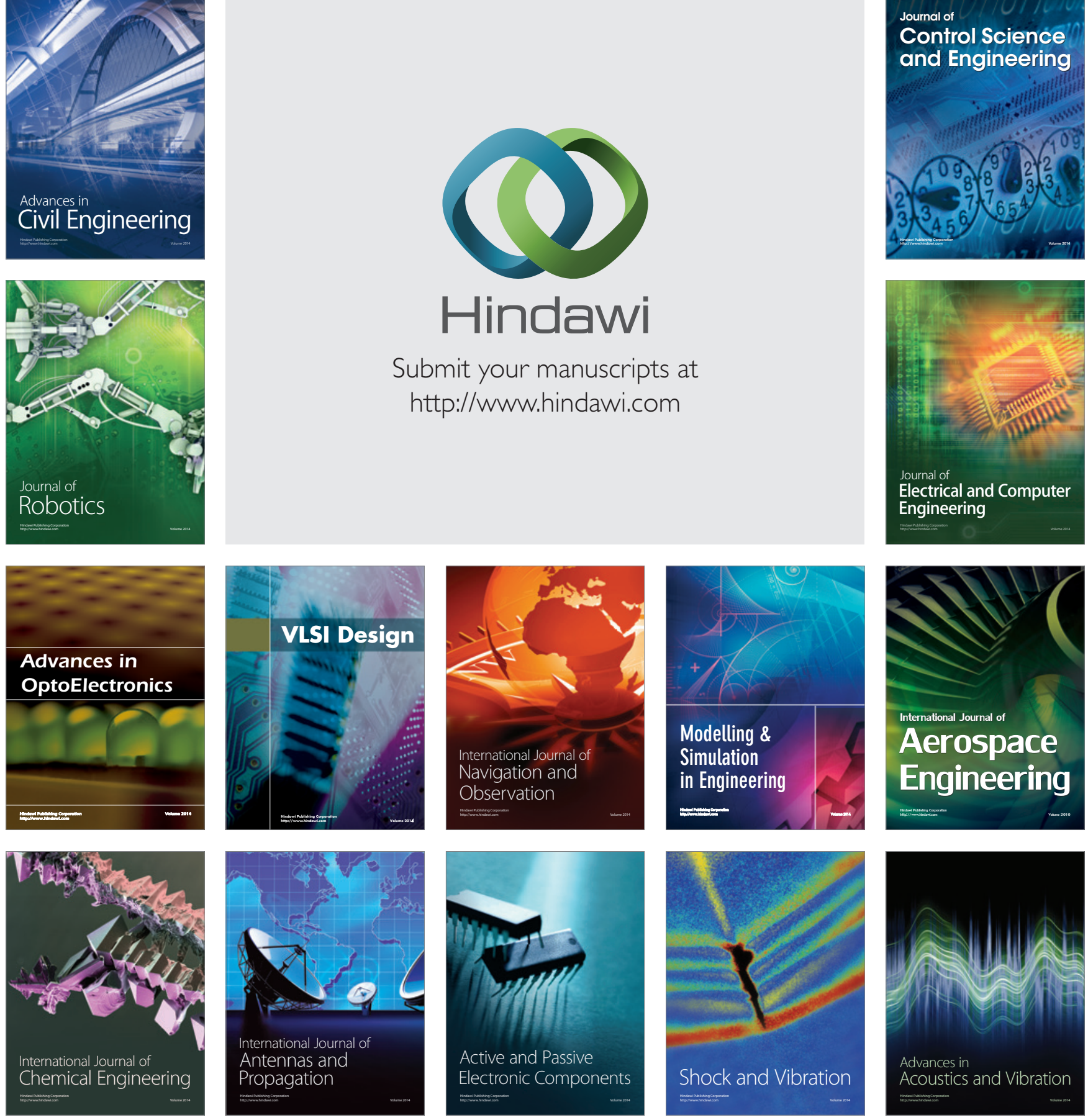